\title{
The calculation of the spatial spectrum of multidimensional fractals using the fast Fourier transform
}

\author{
O.A. Mossoulina ${ }^{1}$

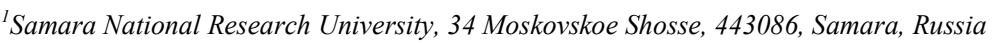

\begin{abstract}
The Fast Fourier Transform was applied to spatial spectrum modeling of a one-dimensional fractal (Cantor set), a two-dimensional fractal (Sierpinski carpet), and a three-dimensional fractal (Menger sponge). A spectrum is developed for different levels. The spatial spectrum was also obtained and modeled for various filling parameters. The ParaView software package was used for 3D modeling.
\end{abstract}

Keywords: cantor set; Sierpinski carpet; Sierpinski carpet; fast Fourier transform; 3D modeling

\section{Introduction}

Many natural phenomena have distinctive features, which are often associated with fractal structures. Visually, fractals represent a geometric figure, replication of which is exactly the same at every scale [1]. This ability is called self-similarity. Fractals are interesting because of widespread presence in natural formations [1-3]. In this case, natural fractals are called "statistical", and artificial "exact". Statistical fractals can be observed in various polymers, biological structures, electrical circuits, galactic clusters and fluctuations in exchange prices [4]. Exact fractals are generated from mathematical approach [5]. Can these precise mathematical abstractions be found in physical reality? Yes, it is optical fractals [3]. This concept includes "diffractals" (diffraction pattern on fractal lattice) [6,7], eigen modes of unstable resonators [8], distributions in nonlinear optics $[3,9]$.

Particularly interesting can be the coincidence of certain properties of "accurate" and "statistical" fractals [10], such as aerosols, smoke, moire [11-13], which is very important applied to optical signal transmission through a heterogeneous or random medium [14-17]. Examination of diffraction on fractal lattice [6, 7, 18-20] can solve other important problems - the formation of periodically self-reproducing fields [21-26], the creation of multi focus [27-30] or specified longitudinal distributions [31-33], and in achromatic depicting systems [34-37].

One of the most important characteristics of fractals is the spatial spectrum [38-41], which are also important in the analysis of crystal structures [42-44]. Taking into account possible multidimensionality of fractals, the calculation of the spatial spectrum can lead to problems associated with computational complexity, which depends on the technical capabilities of modern computers. The solution to the problem can be the usage of the fast calculation algorithm. Within this paper, the fast transformation is used to develop the spatial spectrum of multidimensional fractals with different characteristics.

\section{The calculation of the spatial spectrum of multidimensional fractals}

The first stage of the modeling is the implementation of a one-dimensional case. We take a unit segment $E_{0}=[0,1]$. The next segment is formed according to the rule $E_{1}=[0, a] \cup[b, 1]$, where $a$ and $b$ are the fractal parameters specified in the range of $(0,1)$, whereby $a<b$ and $a+b=1$. We continue until reaching the desired order of the fractal. The intersection of all segments will be a simulated fractal.

$$
E={ }_{i=1}^{n} E_{i},
$$

where $n$ is the order of the fractal.

If the parameters are set to $a=\frac{1}{3}$ and $b=\frac{2}{3}$, then we get the Cantor set.

For programming is used a vector consisting of units, which is successively filled with zeros, according to input parameters and order.

To simulation for two-dimensional case, we used a similar implementation with some corrections. We took the unit square $E_{0}=[0,1] \times[0,1]$ and the next one will take form of $E_{1}=\left(\left[0, a_{1}\right] \cup\left[b_{1}, 1\right]\right) \times\left(\left[0, a_{2}\right] \cup\left[b_{2}, 1\right]\right)$, where $a_{1}, a_{2}, b_{1}$ and $b_{2}$ are fractal parameters specified in the range of $(0,1)$, whereby $a_{1}<b_{1}, a_{2}<b_{2}$ and $a_{1}+b_{1}=1, a_{2}+b_{2}=1$. The simulated fractal can be found by the previously applied for the one-dimensional case formula (1). If we set the parameters $a_{1}=\frac{1}{3}, a_{2}=\frac{1}{3}, b_{1}=\frac{2}{3}$ and $b_{2}=\frac{2}{3}$ we get a fractal called the Sierpinski carpet (Fig. 1). 


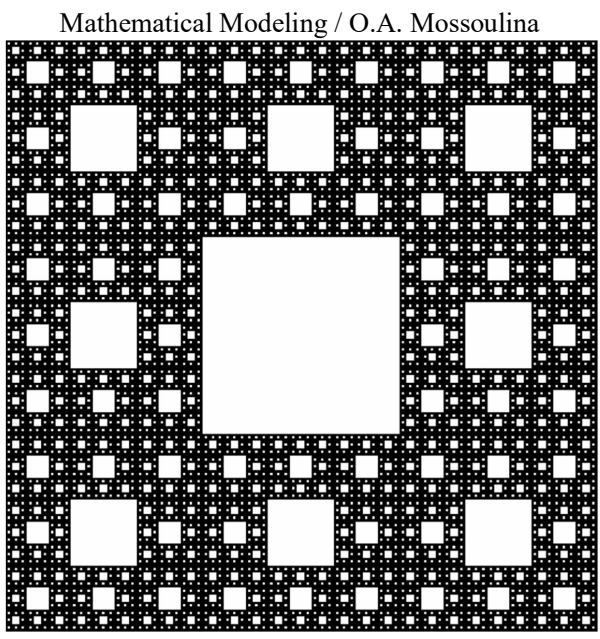

Fig. 1. Fractal (Sierpinsky carpet).

The three-dimensional case is implemented reciprocally to the two-dimensional case. The unit cubes $E_{0}=[0,1] \times[0,1] \times[0,1]$ and $E_{1}=\left(\left[0, a_{1}\right] \cup\left[b_{1}, 1\right]\right) \times\left(\left[0, a_{2}\right] \cup\left[b_{2}, 1\right]\right) \times\left(\left[0, a_{3}\right] \cup\left[b_{3}, 1\right]\right)$ was taken, whereby $a_{1}, a_{2}, a_{3}, b_{1}, b_{2}$ and $b_{3}$ are fractal parameters specified in the range of $(0,1)$, whereby $a_{1}<b_{1}, a_{2}<b_{2}, a_{3}<b_{3}$ and $a_{1}+b_{1}=1, a_{2}+b_{2}=1, a_{3}+b_{3}=1$. If we set the parameters $a_{1}=\frac{1}{3}, a_{2}=\frac{1}{3}, a_{3}=\frac{1}{3}, b_{1}=\frac{2}{3}, b_{2}=\frac{2}{3}$ and $b_{3}=\frac{2}{3}$ we get a three-dimensional fractal called Menger sponge (Fig. 2 a), the boundary section of which is a Sierpinsky carpet.

If we set the parameters $a_{1}=\frac{1}{3}, a_{2}=\frac{3}{8}, a_{3}=\frac{1}{3}, b_{1}=\frac{2}{3}, b_{2}=\frac{5}{8}$ and $b_{3}=\frac{2}{3}$ we get a scalable three-dimensional fractal (Fig. 3 a).

a)

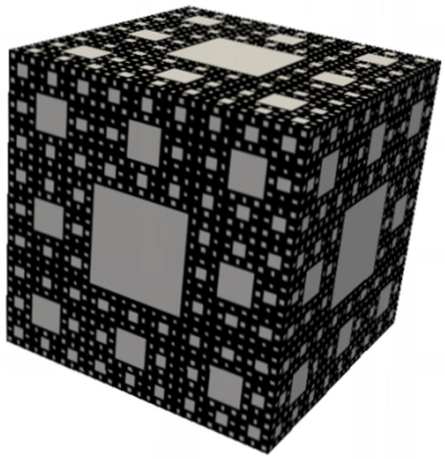

b)

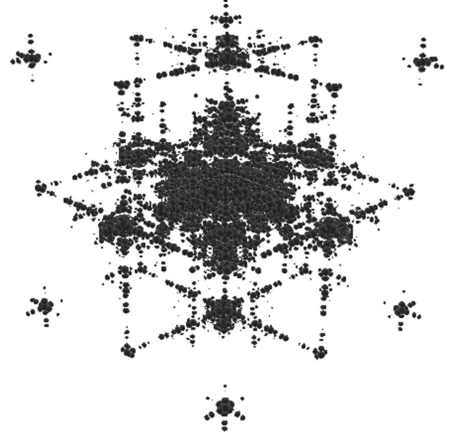

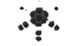

Fig. 2. a) Three-dimensional fractal (Menger sponge), b) the spatial spectrum of a three-dimensional fractal.

a)

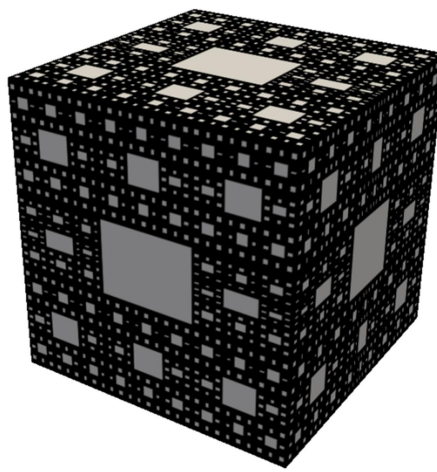

b)

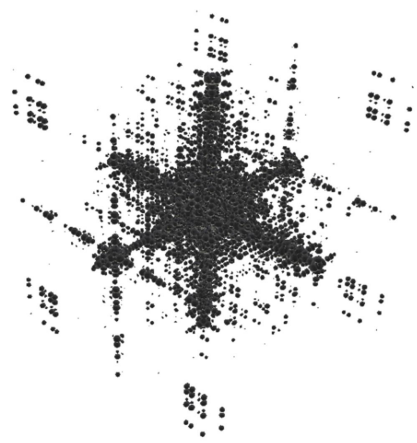

Fig. 3. a) Three-dimensional scalable fractal (Menger sponge), b) the spatial spectrum of a three-dimensional scalable fractal. The Fast Fourier Transform was used to generate the spatial spectrum.

$$
F(\mathbf{u})=\mathfrak{I}[f(\mathbf{x})](\mathbf{u})=\int_{R^{n}} f(\mathbf{x}) \exp (-2 \pi i \mathbf{x u}) d^{n} \mathbf{x},
$$

whereby $f(\mathbf{x})$ is the input function specified as a vector, which is a binary representation of the fractal,

$F(\mathbf{u})$ is the output function,

$\mathfrak{I}[\cdot] \quad$ is the Fourier transform operator. 
The spatial spectrum was obtained from a two-dimensional fractal structure (Sierpinski carpet). The results for the different number of iterations and scale are presented in Table 1.

Table 1.Variability of the spectrum in relations to the number of iterations and scale.

Number

of

Fractal

Spectrum

Fractal

Spectrum

iterations
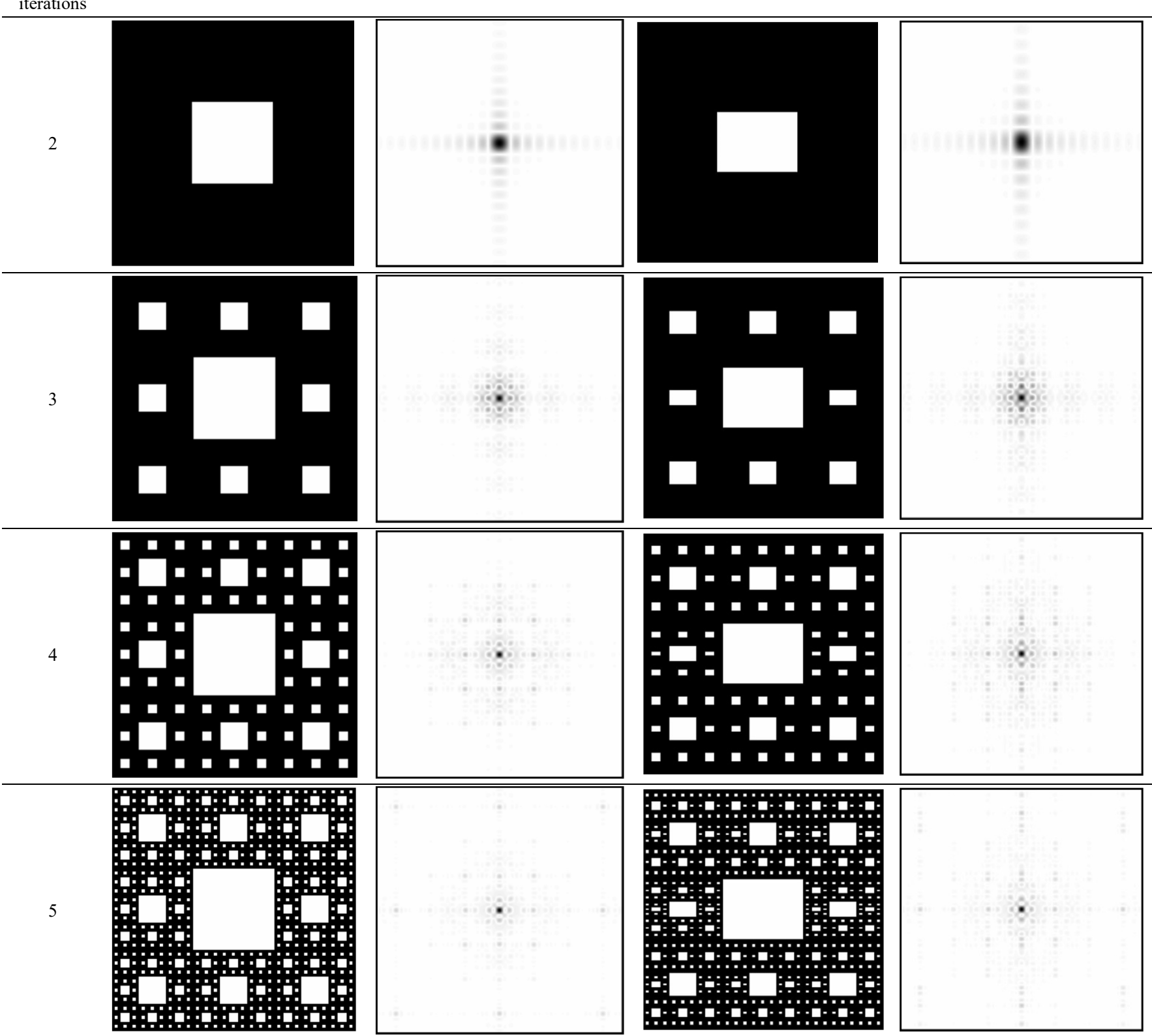

As can be seen from the Table 1, with the number of iteration increasing, the spatial spectrum from the fractal structure becomes more complex and the energy at higher frequencies increases. However, the pattern of the spectrum maintains a regular structure, which is also characteristic of crystalline structures [42-44].

\section{Conclusion}

As a result of the work, the spatial spectrum was calculated and visualized from a two-dimensional (Sierpinski carpet) and a three-dimensional (Menger sponge) fractal structure using the Fast Fourier Transform algorithm.

\section{Acknowledgements}

The work was supported by the Ministry of Education and Science of the Russian Federation.

\section{References}

[1] Mandelbrot BB. The Fractal Geometry of Nature. New York: W.H. Freeman and Company, 1983; 468 p.

[2] Barnsley M. Fractals Everywhere. Academic. Boston: Mass, 1988; 534 p.

[3] Segev M, Soljacic M, Dudley JM. Fractal optics and beyond. Nature Photonics 2012; 6(4): 209-210.

[4] Addison PS. Fractals and Chaos. An illustrated course IOP, 1997; 256 p.

[5] Feder J. Fractals. New York: Springer Science \& Business Media, 2013; 282 p.

[6] Berry MV. Diffractals. J. Phys. A: Math. Gen. 1979; 12: 781-797. 
[7] Berry MV, Klein S. Integer, fractional and fractal Talbot effects. J. Mod. Opt. 1996; 43(10): 2139-2164.

[8] Karman GP, McDonald GS, New GHC, Woerdman JP. Laser optics: Fractal modes in unstable resonators. Nature 1999; 402(6758): 138 p.

[9] Gabitov IR, Manakov SV. Propagation of Ultrashort Optical Pulses in Degenerate Laser Amplifiers. Phys. Rev. Lett. 1983; 50(7): 495 p.

[10] Peitgen HO, Jurgens H, Saupe D. Chaos and fractals: new frontiers of science. 2nd edn. New York: Springer, $2004 ; 864$ p.

[11] Forrest S, Witten TA. Long-range correlations in smoke-particle aggregates. J. Phys. A. 1979; 12(5): L109 p.

[12] Berry MV, Percival IC. Optics of fractal clusters such as a smoke. Journal of Modern Optics 1986; 33(5): $577-591$.

[13] Oster G, Wasserman M, Zwerling C. Theoretical interpretation of moire patterns. J. Opt. Soc. Am. 1964; 54(2): 169-175.

[14] Striletz AS, Khonina SN. Matching and study of methods based on differential and integral operators of laser propagation in media with small inhomogeneities. Computer Optics 2008; 32(1): 33-38.

[15] Khonina SN, Golub I. Creating order with the help of randomness: generating transversely random, longitudinally invariant vector optical fields. Optics Letters 2015; 40(17): 4070-4073.

[16] Soifer VA, Korotkova O, Khonina SN, Shchepakina EA. Vortex beams in turbulent media. Computer Optics 2016; 40(5): 605-624. DOI: 10.18287/24126179-2016-40-5-605-624.

[17] Porfirev AP, Kirilenko MS, Khonina SN, Skidanov RV, Soifer VA. Study of propagation of vortex beams in aerosol optical medium. Applied Optics 2017; 56(11): E8-E15.

[18] Sakurada Y, Uozumi J, Asakura T. Fresnel diffraction by 1-D regular fractals. Pure Appl 1992; 1: 29-35.

[19] Jaggard AD, Jaggard DL. Cantor ring diffractals. Opt. Commun. 1998; 158(1): 141-148.

[20] Szwaykowski P. Self-imaging in polar coordinates. J. Opt. Soc. Am. A 1988; 5(2): 185-191.

[21] Hou B, Xu G, Wen W, Wong GKL. Diffraction by an optical fractal grating. Appl. Phys. Lett. 2004; 85(25): 6125-6127.

[22] Mendez DC, Lehman M. Talbot effect with Cantor transmittances. Optik 2004; 115(10): 439-442.

[23] Kotlyar VV, Soifer VA, Khonina SN. An algorithm for the generation of laser beams with longitudinal periodicity: rotating images. Journal of Modern Optics 1997; 44(7): 1409-1416.

[24] Khonina SN, Kotlyar VV, Soifer VA. Light beams with periodic properties. Methods for Computer Design of Diffractive Optical Elements. Ed. Soifer VA. New York: Wiley \& Sons, Inc., 2002; 535-605.

[25] Almazov AA, Khonina SN. Periodic self-reproduction of multi-mode laser beams in graded-index optical fibers. Optical Memory and Neural Networks 2004; 13(1): 63-70.

[26] Khonina SN, Volotovsky SG. Self-reproduction of multimode laser fields in weakly guiding stepped-index fibers. Optical Memory and Neural Networks 2007; 16(3): 167-177.

[27] Saavedra G, Furlan WD, Monsoriu JA. Fractal zone plates. Optics Letters 2003; 28(12): 971-973.

[28] Mihailescu M, Preda AM, Sobetkii A, Petcu AC. Fractal-like diffractive arrangement with multiple focal points. Opto-electronics review 2009; 17(4): 330337.

[29] Kotlyar VV, Khonina SN, Soifer VA. Diffraction computation of focusator into longitudinal segment and multifocal lens. Proccedings of SPIE 1993; 1780: 263-272.

[30] Soifer VA, Doskolovich LL, Kazanskiy NL. Multifocal diffractive elements. Optical Engineering 1994; 33(11): 3610-3615.

[31] Khonina SN, Kotlyar VV, Soifer VA. Calculation of the focusators into a longitudinal linesegment and study of a focal area. Journal of Modern Optics 1993; 40(5): 761-769.

[32] Khonina SN, Ustinov AV. Design lenses forming paraxial longitudinal distribution according to their spatial spectra. Computer Optics 2013; 37(2): 193202.

[33] Khonina SN, Ustinov AV. Lenses to form a longitudinal distribution matched with special functions. Optics Communications $2015 ; 341$ : $114-121$.

[34] Wang YX, Yun WB, Jacobsen C. Achromatic Fresnel optics for wideband extreme-ultraviolet and X-ray imaging. Nature 2003; $424(6944)$ : 50-53.

[35] Furlan WD, Saavedra G, Monsoriu JA. White-light imaging with fractal zone plates. Opt. Lett. 2007; 32(15): 2109-2111.

[36] Andersen G, Tullson D. Broadband antihole photon sieve telescope. Phys. R. A 2007; 46(18): 3706-3708.

[37] Khonina SN, Ustinov AV, Skidanov RV, Morozov AA. Comparative study of the spectral characteristics of aspheric lenses. Computer Optics 2015; 39(3): 363-369. DOI: - 10.18287/0134-2452-2015-39-3-363-369.

[38] Allain C, Cloitre M. Spatial spectrum of a general family of self-similar arrays. Phys. Rev. 1987; 36(12): $5751-5757$.

[39] Uozumi J, Kimura H, Asakura T. Fraunhofer diffrac-tion by Koch fractals. J. Mod. Opt. 1990; 37(6): 1011-1031.

[40] Zunino L, Garavaglia M. Fraunhofer diffraction by cantor fractals with variable lacunarity. J. Mod. Opt. 2003; 50(5): $717-727$.

[41] Horvath P, Smid P, Vaskova I, Hrabovsky M. Koch fractals in physical optics and their Fraunhofer diffraction patterns. Optik 2010 ; 121 (2): 206-213.

[42] Dal Negro L, Boriskina SV. Deterministic aperiodic nanostructures for photonics and plasmonics applications. Laser Photonics Rev. 2011 ; 1-41.

[43] Kharitonov SI, Volotovskiy SG, Khonina SN, Kazanskiy NL. A differential method for calculating x-ray diffraction by crystals: the scalar theory. Computer Optics 2015; 39(4): 469-479.

[44] Kharitonov SI, Kazansky NL, Volotovskiy SG, Khonina SN. Calculating x-ray diffraction on crystals by means of the differential method. International Society for Optics and Photonics 2016; 10 p. 